\title{
La investigación científica en medicina
}

\author{
Elsa Palou (EP), ${ }^{1}$ Cecilia E. Varela (CV), ${ }^{2}$ \\ Jackeline Alger (JA), ${ }^{3}$ Cristina Rodríguez $(\mathrm{CR})^{4}$
}

\section{CT/ ¿Qué papel juega la investigación científica dentro de las actividades académicas desarrolladas en los posgrados de Medicina? ¿Tiene un papel central? ¿De qué forma?}

EPI Tan central es que si no hacen investigación no se gradúan. Ahora que tengan los estudiantes todo lo que necesitan para hacer investigación, eso es otra cuestión. La situación es que ellos no cuentan con todos los implementos que necesitan para investigar. Para empezar, no disponen de tiempo suficiente para investigar. Luego, no tenemos suficiente recurso humano que los capacite en cómo investigar: no hay suficientes asesores metodológicos y asesores de investigación. Para que ustedes tengan una idea, los cuatros posgrados más grandes: Medicina Interna, Cirugía, Pediatría y Gineco-obstetricia, entre ellos hay cerca de 214 estudiantes y solo 4 docentes contratados para investigación. ¿Pueden 4 personas conducir 214 trabajos de investigación? La respuesta es obvia. Lo que se hace es que con la colaboración de personal que conoce de investigación, como los profesores que están en la Unidad de Investigación Científica en la Facultad de Ciencias Médicas (FCM), también la Dra. Varela que es la

\footnotetext{
Médica internista infectóloga, expresidenta del Colegio Médico de Honduras, exministra de Salud, Premio Internacional de Administración en Salud OPS 2010, miembro del Consejo Técnico Asesor en VIH/SIDA de la OPS, exfundadora y jefe del Servicio de Infectología y Centro de Atención Integral a Pacientes con VIH/SIDA del Instituto Nacional Cardiopulmonar. Actualmente es la coordinadora general de Posgrados FCM/ UNAH, directora de Docencia e Investigación HEU y coordinadora de la Junta Directiva del HEU.

2 Médica especialista en Medicina Interna, especialista en Neumología; egresada de la Policlínica Universitaria de Homburg/Saarland, Alemania; egresada del doctorado en Ciencias Sociales con orientación a la Gestión del Desarrollo, UNAH. Desde 2002, es profesora titular III de los posgrados de la FCM/UNAH. Desde el 2011 es la coordinadora de Investigación en los posgrados de la FCM/UNAH y directora de la Revista Médica de los Posgrados de la FCM.

Médica parasitóloga del Servicio de Parasitología del Departamento de Laboratorio Clínico, HEU. Profesora titular III a medio tiempo de la Unidad de Investigación Científica, FCM/UNAH, desde 2008. Es profesora asociada adjunta al Departamento de Medicina Tropical de la Universidad de Tulane, New Orleans, desde 1997; miembro del consejo editorial de la Revista Médica Hondureña, desde 1998 y de la Revista Médica de los Posgrados en 2012.

${ }^{4}$ Médica con maestría en Salud Pública y diplomada en Gestión de Hospitales. Tiene 28 años de experiencia en hospitales y en salud pública; diplomado en Epidemiología de Campo y Gestión de Programas de Enfermedades Crónicas no Transmisibles. Actualmente es jefe de Unidad de Planeamiento y Evaluación de la Gestión del HEU y miembro del equipo de conducción y gerencia del HEU.
} 
coordinadora de los posgrados que por voluntad propia, no porque estén siendo contratados para eso, sino que por voluntad propia colaboran con los posgrados como asesores metodológicos. De la sesión que vengo, en la cual estaba la jefe del Departamento de Salud Pública, se acaba de dar un listado de 6 personas del Departamento de Salud Pública que van a colaborarnos también como asesores metodológicos.

\section{CT/ ¿Cuáles son los avances que existen en materia de investigación científica en los posgrados de Medicina?}

EP/El año pasado, en el Congreso de los Posgrados, tuvimos 71 egresados que presentaron sus trabajos de investigación; el año anterior, en el 2012, 65 egresados presentaron sus trabajos de investigación. Además, estamos tratando de hacer trabajos intersectoriales y multidisciplinarios que puedan tener mayor impacto en las decisiones políticas en salud.

CV/Desde 1986, los egresados del posgrado en Medicina tienen que presentar, como bien decía la Dra. Palou, un trabajo para poder graduarse. ¿Qué es lo que se ha hecho? Pues yo creo que desde la Universidad, sus políticas han destacado el tema de investigación, se han creado documentos que son las políticas de investigación. La Universidad Nacional Autónoma de Honduras (UNAH) y la FCM, tienen sus documentos de políticas de investigación. Se está trabajando con la Unidad de Investigación Científica en establecer prioridades de investigación con algunos departamentos y especialidades. Todavía no tenemos todo, porque una de las limitantes es cómo ajustar el tiempo de los profesores y estudiantes para el tema de investigación. La mayor actividad es la asistencia, la otra parte del tiempo se da en la academia, la docencia, en dar y preparar clases; y la última, es la parte de la investigación, aunque el tema es central porque sin investigación no se gradúan. El tiempo que tienen ellos para realizarla es limitado. En el plan de mejoras de los posgrados se encuentra el tema de investigación, estamos interesados en que se capacite a los docentes. Otro componente en el plan de mejoras, es el tema de la priorización de las investigaciones; además de la temática de la comunicación de los resultados de las investigaciones y, por último, la bioética. Entonces hemos tenido avances, pero tenemos que organizarnos como unidad, que es lo que está previsto en el reglamento de los posgrados. Está previsto como tal, pero no nos hemos organizado porque hace falta espacio, personas, tecnología, hace falta bastante. Otro avance que se ha dado es el poder trabajar posgrado con el grado en temáticas a nivel nacional, se ha desarrollado un tipo de 
comunicación, facilitada por la Unidad de Investigación Científica, entre residentes y los médicos en servicio social, para orientarles, mientras realizan la investigación, en el diagnóstico y tratamiento de ciertas enfermedades prevalentes, creo que ese es uno de los avances más importantes y que creo que hay que fortalecerlo.

3. CT/ ¿Qué vinculación existe entre la investigación científica y los problemas de salud más frecuentes en la población hondureña?

JA/ En este caso, la vinculación de la investigación y los problemas del país la hemos hecho en una aproximación sistemática a través de un proceso de priorización de la investigación. La FCM tiene priorizadas sus líneas de investigación, también algunos de los departamentos y unidades académicas. Los departamentos y los posgrados más importantes han realizado su proceso de priorización. Entonces, cuando priorizamos, delimitamos lo más importante asignándole un peso a través de criterios, los cuales incluyen aspectos como morbilidad, mortalidad, capacidad de respuesta institucional, entre otros. También se incluyen criterios como dónde vamos a tener más impacto en la población o en los cambios que haya que hacer a nivel institucional. Así, nosotros hemos abordado esta vinculación de manera sistemática y a través de este proceso de priorización.

EPI Para mí, otro avance importante son los trabajos de grupo que han podido realizarse de forma que pueden observarse diversos aspectos de un mismo problema. Por ejemplo, los trabajos que se han hecho en discapacidad marcan pauta y nosotros tenemos una planificación para estudiar problemas reales de salud en Honduras; para el caso, mortalidad perinatal y realizar investigaciones desde el punto de vista del obstetra, del pediatra, del salubrista, de forma que las conclusiones de esos estudios nos permitan tomar decisiones de salud importantes en el hospital y en el país.

4. CT/ ¿De qué manera se articula la atención médica con la investigación científica en salud? ¿Qué ventajas existen? ¿Cómo se pretende aprovechar el Hospital Escuela Universitario para fines de investigación científica?

CR/Entendemos a la investigación científica en salud como un eje transversal en la atención médica. Y se visualiza dentro del plan estratégico institucional y sus objetivos estratégicos para fortalecer esa integración asistencial docente-investigación. Las ventajas son en doble vía, contribuyendo los 
productos de investigaciones científicas con propuestas de mejoras y la calidad de atención a los pacientes en las áreas de curación, rehabilitación, control y en la prevención de la problemática de salud. En este sentido, se pretende aprovechar al Hospital Escuela Universitario (HEU) como un terreno fértil para fortalecer estos procesos de provisión de servicios de salud, a través de las líneas prioritarias de investigación que se han definido acorde a la problemática en salud que está definida en el plan estratégico.

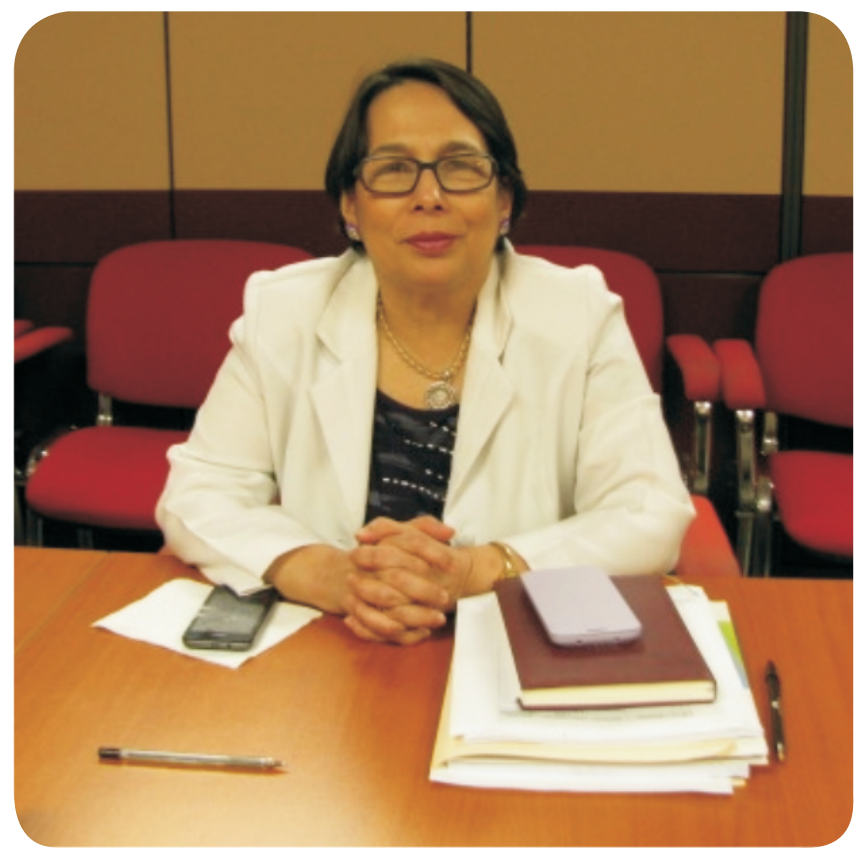

Elsa Palou

5. CT/ En la actualidad, ¿cuáles son las principales investigaciones que se están realizando en el posgrado en el cual usted se encuentra?

CVI Se están realizando investigaciones en los temas, por ejemplo, de tuberculosis, VIH y dengue; también el tema de hipertensión arterial y diabetes, los cuales son temas priorizados por la Secretaría de Salud, es decir que actualmente se les está dando mucha importancia. Además del tema de enfermedades crónicas no transmisibles, y otros como la violencia, lesiones de causa externa, cáncer, por mencionar algunos. 
EP/El trabajo que tuvo el primer lugar el año pasado fue un trabajo sobre cáncer cérvico-uterino. Creemos que los temas prioritarios de los trabajos que se presentan cada año en la FCM están priorizados tanto por la Secretaría de Salud como por la UNAH, tratamos que los trabajos de investigación estén enmarcados en las líneas de investigación de la Facultad y estos, a su vez, con la UNAH. Las líneas de investigación priorizadas de la Facultad de Ciencias Médicas incluyen: 1) Morbi-mortalidad materna infantil, 2) Enfermedades crónicas no transmisibles y estilos de vida, 3) Enfermedades transmisibles y promoción de la salud, 4) Salud mental y violencia, 5) Ambiente, nutrición y salud, 6) Derecho a la salud y los servicios de salud y 7) Gestión académica en la Facultad de Ciencias Médicas. Creemos que estamos enmarcados con las líneas priorizadas por la Universidad como institución, las líneas de la FCM en los principales problemas de salud que tiene el país. Si usted revisa una de las revistas de los posgrados donde están los trabajos de investigación de los residentes, podrá observar que están investigando las patologías prevalentes y más importantes del país.

\section{Desde su punto de vista, ¿cuáles son los principales desafíos que existen actualmente en la investigación científica en el área de salud en el país?}

CVI Yo creo que falta una normativa general a nivel nacional, aunque se ha trabajado con la Organización Panamericana de la Salud en la creación del sistema nacional de investigación en salud y una reglamentación a nivel nacional sobre el tema; por ejemplo, la investigación en sujetos humanos. Actualmente, hay muchas compañías que quieren probar una vacuna y medicamentos y que se hacen a nivel privado, pero en realidad no hay una regulación a nivel nacional en investigación en salud.

EP/No tenemos suficientes comités de bioética. Solo tenemos el de la Unidad de Investigación Científica que tiene que ver con todos los trabajos de investigación que se hacen en el país. Para el caso, la Secretaría de Salud no tiene un comité de bioética. El Hospital ha tenido un comité, pero la función ha sido un poco errática, necesitamos ponerlo a funcionar de una forma más constante. Dependemos básicamente del Comité de Bioética de la Unidad de Investigación Científica. Además, las verdaderas dificultades que tienen los estudiantes son no disponer de tiempo, porque el horario de los posgrados de Medicina son de 16 a 18 horas diarias de trabajo y la mayoría de los posgrados que hacen guardia son de 40 a 42 horas cada tercer día y eso sin sábados, sin domingos, sin feriados. Entonces, no hay suficiente tiempo, no hay suficientes profesores que los asesoren y lo que ellos 
aprenden de investigación en el grado es deficiente. Ellos llevan clases de investigación en salud pública, también tienen clase de Epi Info en salud pública, pero son fragmentadas, no se les da continuidad en los posgrados superiores. Cuando el muchacho llega a los posgrados la verdad es que tiene debilidades en metodología de investigación y el manejo del Epi Info y no tiene el tiempo para ponerse a investigar. Sin embargo, si no investiga no se gradúa. Esta situación les da entender la realidad de la investigación en los posgrados.

JA/ En relación al tema de un sistema nacional de investigación para la salud. Desde mi punto de vista, ese sería un principal desafío en el país, que es conformar ese sistema nacional. En este sistema, por supuesto, la Secretaría de Salud tendría un papel preponderante como regulador. Pero también intervienen otros actores y ahí está la UNAH, Secretaría de Finanzas y Secretaría de Educación, para nombrar algunas. En el sistema intervienen múltiples actores, pero lo principal es contar con un marco regulatorio legal, con financiamiento competitivo, que debería estar muy regulado, en donde con unas reglas y bases transparentes diferentes grupos a nivel nacional puedan participar y competir por fondos nacionales, eso debería estar enmarcado en una prioridad de investigación a nivel nacional. La UNAH tiene prioridades a nivel de investigación, la Secretaría de Salud tiene prioridades a nivel de atención, pero la Secretaría de Salud no ha hecho el ejercicio de identificar prioridades de investigación, estas deben responder a las necesidades de la población y las necesidades institucionales, pero eso sí, se necesita voluntad y un proceso sistemático.

CV/ Sin embargo, esas agencias e instituciones internacionales de cuidado de la salud como la Organización Mundial de la Salud y la Organización Panamericana de la Salud, le están dando mucho énfasis a la investigación y, sobre todo, a lo que es investigación operativa. Ellos necesitan datos de la realidad nacional para poder revisar las estrategias, las políticas y las estrategias de salud precisamente para los países.

EP/Si concluimos en lo que es más práctico, estamos viviendo un problema por la falta de digitadores en la Facultad de Ciencias Médicas, la falta de un centro de cómputo que permita en horarios de fin de semana y horarios nocturnos que los estudiantes de Medicina, que trabajan todo el día, puedan ir a digitar sus trabajos de investigación. $Y$ eso hace que el muchacho se tenga que ir y pagar compañías de digitación que son externas. Entonces, creemos que son cosas bien concretas que facilitarían 
mucho la producción académica y la producción de lo que se está trabajando en investigación en salud.

7. CT/ Desde su experiencia en el posgrado, ¿qué papel juega el docente para incentivar la investigación científica en sus alumnos de posgrado? ¿Qué importancia asigna usted a que el docente de posgrado sea un investigador con amplia experiencia?

EP/ El estudiante tiene que tener una enseñanza en la investigación en forma transversal durante todo su posgrado. Lo cierto es que los estudiantes, antes de entrar a los posgrados, reciben de la Unidad de Investigación Científica un curso de metodología de la investigación científica que es puntual, pero nosotros creemos que lo ideal sería que el estudiante pudiera tener una clase de metodología de la investigación todas las semanas; sin embargo, no hay suficientes docentes capacitados en el tema como para poder hacer eso. Son 19 posgrados clínicos, 4 de Enfermería, 1 de Salud Pública y 1 de Epidemiología. Son un total de 25 posgrados y en la Unidad de Investigación Científica hay 7 docentes. Obviamente, no son suficientes como para poder implementar una enseñanza transversal de la metodología de la investigación.

CVI Uno de los avances ha sido nombrar coordinadores de investigación en la mayoría de los posgrados. Lastimosamente, el tema de capacitación de docentes con alta experiencia o alguna experiencia en metodología de investigación en realidad es difícil de encontrar aquí, igualmente para los que se contratan como médicos asistenciales del Hospital Escuela.

EPI Precisamente, sobre lo que dice la Dra. Varela, tener un coordinador de investigación es lo que se trata de hacer con los posgrados, pero no en todos los posgrados tenemos coordinadores de investigación. Por ejemplo, en el pos-grado de Pediatría tenemos 1 coordinador de investigación y 65 residentes. En Gineco-obstetricia hay 1 coordinador para 56 residentes, en Medicina Interna hay 1 coordinador de investigación para 48 residentes. Entonces, yo veo que en otros posgrados hay un coordinador de investigación para 3, 4 o 7 personas, si está muy cargado. Hay posgrados que son muchos menos, en Cirugía Plástica son como 3 o 4. Hemos hablado con el Departamento de Salud Pública porque creemos que en este hay magísteres que pueden colaborar con los posgrados y nos dijeron que tenían anuencia. Esperamos empezar a tener relación con este Departamento, con los posgrados, porque al final, los magísteres en salud pública 
son profesores de la Facultad y, por tanto, como profesores de la Facultad, deben dar clases en grado y posgrado.

CR/ Otro gran reto sería la retroalimentación a los espacios asistenciales, porque eso podría fortalecer mucho el abordaje de la atención médica, establecer protocolos de atención, porque eso mejora la atención a nuestros pacientes y en eso siento que todavía no se está dando. Se está haciendo mucha investigación, pero los productos no están regresando al ámbito de donde salieron.

EPI Hay unos posgrados que ya lo empezaron a hacer, Medicina Interna y Gineco-obstetricia. Hay varios posgrados que reúnen a los médicos asistenciales de su departamento y al residente, además de presentar su trabajo de investigación en el Congreso de los Posgrados, lo presentan internamente en su departamento y ahí lo discuten. Pero eso es algo que puede ser promovido, y podríamos hacerlo, en sesiones clínicas, con departamentos más grandes en conjunto para hacer discusiones de estas investigaciones. De repente, los hallazgos de estos muchachos pueden cambiar decisiones sobre cómo funciona el Hospital en ciertas áreas.

CVI Es necesario que esta información no pertenezca solamente a los ámbitos académicos. Por ejemplo, cuando estuve dando clase en Medicina Interna, además de las revisiones bibliográficas de la producción internacional, nosotros les pedíamos que revisaran lo que se había publicado aquí en Honduras, particularmente en las residencias. Además, se les enviaba a obtener infor-mación de lo que es el Departamento de Epidemiología, porque sí hay producción académica, pero de repente no se visibiliza suficiente. Yo creo que no es tanto el acceso, creo que estamos acostumbrados a buscar solamente la información que se produce en el exterior.

EPI Y nosotros no socializamos, esa es nuestra culpa. Nosotros presentamos en los congresos de los posgrados, aparece en la revista, pero de ahí no hay una socialización masiva.

8. CT/ En su opinión, ¿de qué manera se puede desarrollar una perspectiva de investigación interdisciplinaria en los posgrados de Medicina con otras áreas del conocimiento?

EP/Es muy factible en la medida que la UNAH lo permita, porque para nosotros 
el problema que encontramos es que se exige al estudiante que su trabajo de graduación sea unipersonal. Si se permitiera que fueran grupales y tener un trabajo en el cual haya gente de psicología, economía y medicina, creo que podríamos incentivar eso.

CV/Es más, en unos espacios se ha discutido sobre la riqueza de los trabajos de tesis a diversos niveles de maestría y doctorado que pudieran ser grupales para fomentar las relaciones entre las diversas instituciones.

JA/ En el marco de las líneas prioritarias de investigación de la Facultad hay una posibilidad real de realizar investigaciones interinstitucionales y multidisciplinarias. La mayoría de las investigaciones interinstitucionales que hemos realizado han sido con nuestro aliado estratégico natural, que es la Secretaría en Salud, junto con los hospitales y luego las unidades de la Red de Salud. Entonces, tenemos ejemplos de estudios como el de Prevalencia de demencia y factores asociados en adultos mayores en las áreas de influencia de los médicos en servicio social. Además, la FCM estaba participando con la Asociación Hondureña de Alzheimer, pediatras del Instituto Hondureño de Seguridad Social y salubristas del Programa Nacional del Adulto Mayor de la Secretaría de Salud, lo cual se nos facilita por los horarios y los temas que la investigación interinstitucional y multidisciplinaria desarrolla con instituciones como estas. Para hacer una investigación interdisciplinaria con otras facultades de la UNAH, necesitaríamos una facilitación a través de la Dirección de Posgrados. Y definitivamente, se debe mejorar la relación docente-estudiante, que actualmente está desbalanceada por el escaso número de docentes y el mayor número de estudiantes. Para facilitar ese tipo de investigación se requiere de tiempo real que tenga el docente para hacer a esa gestión y tiempo es lo que menos se tiene.

CR/ En este momento, desde la Unidad de Planeamiento estamos haciendo investigaciones operativas, trabajamos con dos licenciados en economía que nos están apoyando en un proyecto de investigación sobre la carga social y económica que nos está dando determinada morbilidad en el Hospital. También estamos trabajando con ingeniería en biomédica, la que nos está haciendo una investigación para fortalecer proyectos de gestión e intervención para obtener insumos para que se hagan proyectos en el manejo hospitalario, que es un problema importante que tenemos en nuestro Hospital. Asimismo, llegó una licenciada en psicología y otra en trabajo social para hacernos unos estudios socioeconómicos para hacer 
una venta de servicios. Hemos estado haciendo gestiones para que otras disciplinas en investigación se relacionen con la parte médica.

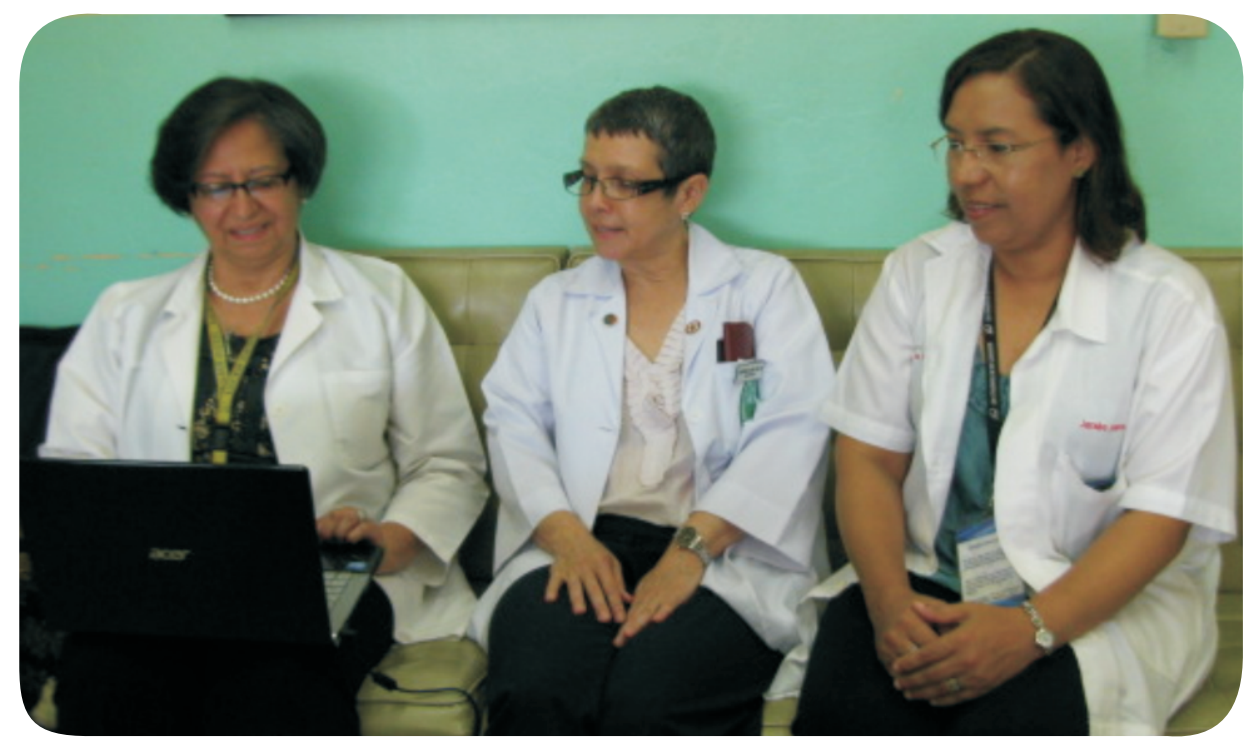

Cecilia Varela, Jackeline Alger, Cristina Rodríguez

\section{CT/ ¿Cuáles son los esfuerzos que se están realizando desde la Facultad} de Medicina para adaptarse a las tendencias internacionales en investigación científica en el área de salud? ¿Cuáles son los avances? ¿Dónde se encuentran los retos?

JA/Es importante tener en cuenta el aspecto de capacitación y certificación de los investigadores, tanto estudiantes de grado y posgrado como docentes, en ética de la investigación. Nosotros tenemos varios años, a través de la Unidad de Investigación Científica y los Posgrados de la FCM, en promocionar y regular la capacitación y certificación de todos los investigadores en ética en investigación y para eso tenemos dos colaboradores internacionales. Uno es el programa Colaborative Institutional Training Iniative (CITI) de la Universidad de Miami; y el otro es una es una institución llamada The Global Health Network, ambas nos proporcionan cursos en línea sobre temas como buenas prácticas clínicas, conducta responsable en la investigación, protección de sujetos humanos en la investigación e integridad científica. Con estos temas se cubren los módulos y ellos al final dan una lista de los módulos tomados por los estudiantes y los docentes. Este es un requisito que se les pide tanto a los estudiantes de Medicina en su último 
año de carrera, como a los estudiantes de posgrados en los diferentes semestres. Con esta capacitación esperamos que se les prepare mejor para el desarrollo de las investigaciones.

EP/Sé que la Facultad tiene convenios con la Universidad de Columbia, también con algunas universidades de Alemania, con la Universidad de Sao Paulo, con la Universidad de México y sé que trabaja con algunas universidades en Inglaterra. Lo que necesitamos es ver cómo eso se concretiza en trabajos de investigación multilaterales o bilaterales, porque hasta ahora es más una relación de tipo administrativo y gerencial, pero necesitamos que eso baje a nivel operativo y que se tenga un trabajo operativo de investigación.

CR/ En el tema de trauma y violencia, desde el año pasado la Dirección de Docencia e Investigación hizo contactos con la Universidad de Harvard y ya están en proceso; de hecho, ellos abrieron más espacio para la relación para mejorar la articulación del Hospital con instituciones universitarias de prestigio internacional, lo cual fortalece el ámbito de la investigación.

EP/ Ya hay con esa Universidad un trabajo colaborativo en temas de violencia y atención de víctimas de la violencia en el Hospital y tenemos otras universidades que han mostrado interés como Hospital. Creo que la ventaja que tienen actualmente los posgrados es que la UNAH tiene la administración del Hospital y de los posgrados. Entonces, se pueden hacer este tipo alianzas desde ambas entidades, como Hospital y como posgrado 